\title{
FKBP5 Genotype and Structural Integrity of the Posterior Cingulum
}

\author{
Negar Fani*,', Tricia Z King ${ }^{2}$, Emily Reiser', Elisabeth B Binder ${ }^{1,3}$, Tanja Jovanovic', Bekh Bradley ${ }^{4}$ and \\ Kerry J Ressler ${ }^{1,5}$ \\ 'Department of Psychiatry and Behavioral Sciences, Emory University School of Medicine, Atlanta, GA, USA; ${ }^{2}$ Department of Psychology, Georgia \\ State University, Atlanta, GA, USA; ${ }^{3}$ Max Planck Institute of Psychiatry, Munich, Germany; ${ }^{4}$ Atlanta VA Medical Center, Decatur, GA, USA; \\ ${ }^{5}$ Howard Hughes Medical Institute, Chevy Chase, MD, USA
}

\begin{abstract}
Alterations in the microarchitecture of the posterior cingulum (PC), a white matter tract proximal to the hippocampus that facilitates communication between the entorhinal and cingulate cortices, have been observed in individuals with psychiatric disorders, such as depression and post-traumatic stress disorder (PTSD). PC decrements may be a heritable source of vulnerability for the development of affective disorders; however, genetic substrates for these white matter abnormalities have not been identified. The FKBP5 gene product modulates glucocorticoid receptor function and has been previously associated with differential hippocampal structure, function, and affect disorder risk. Thus, FKBP5 is an attractive genetic target for investigations of PC integrity. We examined associations between PC integrity, measured through diffusion tensor imaging (DTI) and fractional anisotropy (FA; an index of white matter integrity), and polymorphisms in the FKBP5 SNP rs/360780 in a sample of 82 traumatized female civilians. Findings indicated that, compared with individuals without this allele, individuals who carried two 'risk' alleles for this FKBP5 SNP (T allele; previously associated with mood and anxiety disorder risk) demonstrated significantly lower FA in the left PC, even after statistically controlling for variance associated with age, trauma exposure, and PTSD symptoms. These data suggest that specific allelic variants for an FKBP5 polymorphism are associated with decrements in the left PC microarchitecture. These white matter abnormalities may be a heritable biological marker that indicates increased vulnerability for the development of psychiatric disorders, such as PTSD.
\end{abstract}

Neuropsychopharmacology (2014) 39, 1206-1213; doi:10.1038/npp.2013.322; published online I8 December 2013

Keywords: FKBP5; MRI; DTI; posterior cingulum; hippocampus; anxiety

\section{INTRODUCTION}

A growing number of studies document disruptions in white matter architecture in individuals with mood and anxiety disorders using diffusion tensor imaging (DTI) methods (Thomason and Thompson, 2011; Fani et al, 2012; Tromp do et al, 2012). Many of these studies highlight degraded integrity of connections between gray matter structures that are responsible for emotion regulation and executive control. In particular, alterations in functional connectivity between frontal/anterior cingulate cortex (ACC) and limbic regions, such as the amygdala and hippocampus, have characterized depressed and anxious individuals (Sylvester et al, 2012; Zeng et al, 2012; Zhang et al, 2011). The cingulum is a white matter tract that connects the entorhinal cortex and the cingulate cortex, facilitating communication between the ACC and hippocampus. Damage to

*Correspondence: Dr N Fani, Department of Psychiatry and Behavioral Sciences, Emory University, 101 Woodruff Circle, Suite 4304, Atlanta, GA 30322, USA, Tel: +404 727 8265, Fax: +404 778-। 488,

E-mail: nfani@emory.edu

Received 27 August 2013; revised 27 October 2013; accepted II November 2013; accepted article preview online 20 November 2013 this pathway is likely to disrupt cognition and/or emotion regulation; in combination with other predisposing factors, abnormalities in this white matter tract may significantly increase vulnerability for the development of mood and anxiety disorders.

In particular, structural abnormalities in posterior aspects of the cingulum (PC), assayed with DTI, may promote emotional disturbance; these abnormalities have been highlighted in DTI studies of psychiatric disorders, including schizophrenia (Chen et al, 2013; Fujiwara et al, 2007), anorexia (Kazlouski et al, 2011), depression (Schermuly et al, 2010), bipolar disorder (Paillere Martinot et al, 2013), and post-traumatic stress disorder (PTSD; Fani et al, 2012). Abnormalities in PC integrity have also been identified in traumatized populations, including individuals who experienced childhood abuse (Choi et al, 2009), natural disasters (Wang et al, 2010), and interpersonal trauma (Fani et al, 2012). The PC is a white matter pathway proximal to the hippocampus, providing the main route of communication to frontal regions, facilitating efficient integration of emotional and cognitive information. Disruptions in this region are likely to adversely influence both affective and cognitive processes. Regarding cognition, degraded PC microarchitecture has been linked to Alzheimer's disease (Zhang et al, 
2007), mild cognitive impairment (Delano-Wood et al, 2012; Drago et al, 2011), and poorer performance on measures of selective attention, executive function (Schermuly et al, 2010), and memory (delayed recall; Kantarci et al, 2011), illustrating the salience of PC integrity in higher-order executive processes. Taken together, these findings suggest that PC disruptions have consequences for processing of incoming emotional and cognitive information.

These PC abnormalities may be associated with particular genetic profiles and could represent a heritable source of vulnerability for the development of affective and cognitive disorders. Mounting evidence suggests that the development of white matter architecture is largely under the influence of genetic traits (Chiang et al, 2009; Kochunov et al, 2010); however, the genetic contributions to specific white matter pathways, including the cingulum, have not been identified. Clarifying associations between PC disruptions and genetic profiles represents an important step toward identifying white matter signs of vulnerability for the development of psychiatric disorders, such as PTSD.

FKBP5 is a particularly attractive genetic target for investigations of PC integrity given its putative function and location of expression. The FKBP5 gene is known to have a role in stress regulation by modulating the sensitivity of a receptor (the glucocorticoid receptor (GR)) (Binder, 2009). The GR is central to the processes underlying acute and chronic stress, providing feedback to the central stress regulatory system. The hippocampus is a brain region that is densely populated with GRs (Morimoto et al, 1996), demonstrates high FKBP5 mRNA expression (Scharf et al, 2011), and similarly is highly vulnerable to the effects of stress. Increased circulating levels of glucocorticoids have been associated with significantly decreased cell proliferation in the dentate gyrus of the hippocampus and surrounding white matter (Alonso, 2000). Abnormalities in hippocampal function (Fani et al, 2013) and volume (Zobel et al, 2010) have been linked to FKBP5 genetic status and are frequently manifest in psychiatric disorders that may be influenced by this gene, such as PTSD (Bremner et al, 1995; Gurvits et al, 1996; Bremner et al, 2003; Acheson et al, 2012; Shin and Liberzon, 2010). Among other FKBP5 SNPs, rs1360780 SNP is of particular interest, given its putative functionality and associations with psychopathology (Binder et al, 2008; Lavebratt et al, 2010; Mehta et al, 2011). A haplotype in this gene locus has been shown to interact with child abuse to predict risk not only for PTSD but also for depression, aggression, and psychosis (Binder et al, 2008; Lavebratt et al, 2010; Mehta et al, 2011; Bevilacqua et al, 2012; Collip et al, 2013). The most likely variant conferring the functional differences seen with this haplotype is rs1360780, located in intron 2 of the FKBP5 locus in close proximity to a functional glucocorticoid response element (GRE). A recent finding indicates that the risk allele of this SNP leads to a different 3D conformation of the DNA, likely by enhanced binding to TATA-box binding protein, bringing the GRE in intron 2 in physical contact with the transcription start site and leading to an enhanced stimulation of FKBP5 mRNA by glucocorticoids (Klengel et al, 2013). This altered molecular feedback between GR activation and FKBP5 has been shown to impact GR function and by extension the stress hormone response (Binder et al, 2008; Ising et al, 2008; Luijk et al, 2010). Given these data, it seems probable that FKBP5 would affect GR functioning within the hippocampus and thus also influence the integrity of white matter tracts proximal to the hippocampus, namely, the posterior cingulum.

Recently, we conducted a voxel-wise analysis investigating differences in white matter tract integrity between individuals with PTSD and similarly traumatized controls without this disorder (Fani et al, 2012). Our findings revealed that individuals with PTSD demonstrated lower fractional anisotropy (FA; a scalar index indicating the strength of diffusion direction within white matter tracts) within the PC, particularly in the left hemisphere, compared with traumatized individuals without PTSD. Given that both groups experienced similar levels of trauma exposure, and in light of evidence suggesting that PTSD is partly heritable (with heritability estimates of approximately $40 \%$; reviewed in Almli et al, 2013), we hypothesized that genetic substrates may have influenced the development of these white matter abnormalities, possibly after exposure to major stressors.

The present study was designed to investigate the possibility that FKBP5 genetic status differentially influences microstructural integrity of the PC. We examined whether particular FKPBP5 genotypes would be associated with decrements in PC integrity, a biological phenotype that may modulate risk for the development of anxiety disorders, such as PTSD. We hypothesized that the presence of 'risk' alleles for FKBP5 genotype would be associated with significantly lower FA in the PC after statistically controlling for factors that may influence FA, including age, trauma exposure, and PTSD symptoms. Gene by environment (ie, history of childhood maltreatment) interactions were also tested for FKBP5 genotype, given the relevance of this interaction in earlier PTSD association studies (Binder et al, 2008; Mehta et al, 2011; Klengel et al, 2013; Boscarino et al, 2012; Xie et al, 2010). Analyses were conducted with a putatively functional FKBP5 SNP, rs1360780 (Klengel et al, 2013), similar to a recent study that found associations of this SNP with hippocampal structure and function (Fani et al, 2013).

\section{MATERIALS AND METHODS}

\section{Participants}

A total of 82 African-American women between 18 and 62 years of age were enrolled as a part of a larger study investigating genetic risk for stress-related disorders; data from 51 participants from an earlier published study (Fani et al, 2012) were included. Race was self-reported. Study procedures were approved by the institutional review board of Emory University School of Medicine. Participants were recruited from the general medical clinics of a publicly funded hospital, as detailed previously (Bradley et al, 2008). Individuals were deemed eligible for participation if they could give informed consent and understand English, as determined by a study researcher. Participants were administered the Traumatic Events Inventory (TEI) to detail frequency and type of traumatic experiences and the PTSD Symptom Scale (PSS; Falsetti et al, 1993), to measure the presence and frequency of current PTSD symptoms. We also used this measure to classify individuals as controls (C) or participants with PTSD $(\mathrm{PTSD}+$ ) based on DSM-IV 
criteria, consistent with our earlier study (Fani et al, 2012). The Childhood Trauma Questionnaire (CTQ) was used to measure trauma exposure during childhood, specifically, maltreatment. The CTQ is a 28 -item self-report measure (Bernstein and Fink, 1998) that yields indices of sexual, physical, and emotional abuse, which may be combined for a total score. Demographic and clinical characteristics of this sample, stratified by genotype for FKBP5 SNP rs1360780, are detailed in Table 1.

Exclusion criteria for this study included current psychotropic medication use, medical, or physical conditions that preclude MRI scanning; a history of bipolar disorder, schizophrenia, or other primary psychotic disorder; current alcohol or substance abuse; medical conditions that contribute significantly to psychiatric symptoms; history of head injury or loss of consciousness for $>5 \mathrm{~min}$; or history of neurological disease.

\section{DNA Extraction, Genotyping, and Quality Control}

The FKBP5 SNP rs1360780 was selected based on our previous studies of FKBP5 polymorphisms within this population (Binder et al, 2008; Mehta et al, 2011). Using an additive model, participants were categorized into three

Table I Demographic and Clinical Characteristics

FKBP5 SNP rs 1360780

\begin{tabular}{|c|c|c|c|c|}
\hline & $C C(n=28)$ & CT $(n=34)$ & TT $(n=20)$ & \\
\hline & Mean (SD) & Mean (SD) & Mean (SD) & $p$ \\
\hline Age & $43.2(13.4)$ & $35.6(12.3)$ & $37.9(13.3)$ & 0.14 \\
\hline PSS total & $12.8(12.4)$ & $15.2(\mid 1.5)$ & $11.6(10)$ & 0.51 \\
\hline CTQ total & $43(14.6)$ & $41.5(20.1)$ & $38.8(10.3)$ & 0.68 \\
\hline CTQ sexual abuse & $10.1(6)$ & $9.3(6.5)$ & $6.9(3)$ & 0.14 \\
\hline CTQ physical abuse & 8.1 (3) & $8.4(4.8)$ & $7.4(2.1)$ & 0.63 \\
\hline CTQ emotional abuse & $8.5(3.6)$ & $8.8(5.1)$ & $8.9(2.9)$ & 0.94 \\
\hline TEl adult total & $3.9(2.4)$ & $3.4(1.5)$ & $4.5(2.6)$ & 0.21 \\
\hline TEl total & $4.8(2.2)$ & $3.1(1.6)$ & $2.6(2)$ & 0.52 \\
\hline
\end{tabular}

\begin{tabular}{lcccc}
\hline & $\%$ & $\%$ & $\%$ & $\begin{array}{l}\text { Kruskal- } \\
\text { Wallis } \boldsymbol{p}\end{array}$ \\
& & & & 0.74 \\
\hline Education & & & & \\
$\quad<$ I2th grade & 17.9 & 11.8 & 10 & \\
I2th grade/high school graduate/GED & 17.9 & 32.4 & 45 & \\
Some college/technical school & 39.3 & 35.3 & 25 & \\
Tech school/college graduate & 25 & 20.6 & 20 & \\
& & & & \\
Household monthly income & & & & \\
\$0-249 & 14.8 & 12.5 & 15 & 0.52 \\
\$250-499 & 7.4 & 18.8 & 20 & \\
\$500-999 & 33.3 & 21.9 & 35 & \\
\$1000-1999 & 29.6 & 40.6 & 25 & \\
\$2000+ & 14.8 & 6.2 & 5 &
\end{tabular}

Abbreviations: PSS, PTSD symptom scale; TEl, traumatic events inventor, CTQ, childhood trauma questionnaire.

* $p<0.05$. groups based on genotype, ie, how many 'risk' alleles comprised their genotype, based on previous studies: for this SNP, individuals were categorized as having either one or two copies of the $\mathrm{T}$ allele.

Saliva was collected in Oragene vials (DNA Genotek, Ontario, Canada). DNA was extracted using the Agencourt DNAdvance extraction kit (Beckman Coulter, Danvers, MA) and then quantified using gel electrophoresis and Quantity One software (Bio-Rad, Hercules, CA) or NanoDrop2000 (Thermo Fisher Scientific, Waltham, MA). The DNA was normalized to $10 \mathrm{ng} / \mu \mathrm{l}$, and a total amount of 15 or $20 \mathrm{ng}$ of dried down DNA per reaction was used for Taqman and Sequenom reactions, respectively. Sequenom reactions were performed using iPlex reagents on the MassARRAY system (Sequenom, San Diego, CA). Additional reactions were performed on the Taqman ViiA7 Real-Time PCR system using Taqman SNP Genotyping Assays as well as Taqman Genotyping Master Mix or Taqman GTXpress Master Mix (Life Technologies, Carlsbad, CA).

Alleles passed Hardy-Weinberg Equilibrium at $p>0.05$. The call rates on both platforms were $>95 \%$. Negative controls as well as within-plate and across-platform duplicates were used for quality control. Within the Sequenom genotyping calls, the duplication rate was $2.1 \%$ and within the Taqman genotypes, the duplication rate was $16.3 \%$. Samples were duplicated on both platforms to give an overall across-platform duplication rate of $7.2 \%$. Within these duplications, there were no discordant genotype calls; additionally, no negative controls were called.

\section{MRI Acquisition, Image Processing, and Statistical Analyses}

Scanning took place on a research-dedicated Siemens 3-Tesla TIM-Trio scanner at the Emory University Hospital. Diffusion-weighted images were acquired with maximum gradient strength of $40 \mathrm{~m} / \mathrm{Tm}$ with the following parameters: $39 \times 2.5 \mathrm{~mm}^{2}$ thick axial slices, matrix $=128 \times 128$, field of view $=220 \times 220 \mathrm{~mm}$, voxel size $=1.72 \times 1.72 \times 2.5 \mathrm{~mm}^{3}$. The diffusion weighting was isotropically distributed along 60 directions using a $b$-value of $1000 \mathrm{~s} / \mathrm{mm}^{2}$. Four normalization images, with no diffusion encoding $(b=0)$, were acquired and averaged for each direction using linear rigid body registration (FLIRT; Jenkinson and Smith, 2001). All image processing and analysis was conducted using the FMRIB Software Library FSL version 4.1; www.fmrib.ox. ac.uk/fsl; Smith et al, 2004).

Correction for head motion and eddy current distortion was performed for data from each individual participant using an automated affine registration algorithm. Normalization images were skull-stripped using the FSL brain extraction tool (Smith, 2002). FA maps were generated using the DTIfit in the FMRIB Diffusion Toolbox. Using the tract-based spatial statistical approach available in FSL (TBSS; version 1.2 ;Smith et al, 2006), all participants' FA maps were non-linearly registered to a common space (FMRIB58_FA), then affine transformed into $1 \times 1 \times 1 \mathrm{~mm}^{3}$ MNI space. Transformed FA images were averaged to create a mean FA image, then thinned to create a mean FA skeleton, which represented the centers of all tracts common to the group. This was thresholded by FA $>0.2$ to ensure that gray matter regions would be excluded from 
analyses. Voxel values of each subject's FA map were projected onto the skeleton by searching the local maxima along the perpendicular direction from the skeleton. Masks of the PC were created based on earlier findings (Fani et al, 2012); confirmatory analyses were conducted using masks that were created based on the probabilistic Johns Hopkins White Matter Atlas (Hua et al, 2008) provided in FSL and thresholded by $20 \%$ to reduce the likelihood of including irrelevant tracts (see Figure 1). FA values were extracted from these regions using FSLstats and entered into statistical models.

Univariate ANCOVAs were conducted to examine between-group differences in FKBP5 genotype for left and right PC FA; covariates included age, PTSD symptoms, childhood maltreatment, and adult trauma exposure, given that these factors have been previously been associated with FA (Fani et al, 2012; Choi et al, 2009; Wang et al, 2010; Lebel et al, 2012; Ly et al, 2013). Factorial ANCOVAs with FKBP5 genotype and PTSD diagnosis as factors and age, childhood, and adult trauma as covariates were also conducted to test genotype by diagnosis interactions on PC FA. Factorial ANCOVAs were used to test for interactions between genotype and childhood maltreatment history (presence or absence, as indicated on the CTQ) for left and right PC FA, after controlling for PTSD symptoms, age, and trauma exposure in adulthood. Statistical significance was defined as $p<0.025$ for primary analyses, representing Bonferroni correction for separate tests (left and right PC); a threshold of $p<0.05$ was set for confirmatory analyses conducted with atlas-based masks.

\section{RESULTS}

\section{Group Characteristics}

A total of 82 participants were included in this study. Findings from univariate ANOVAs and Kruskal-Wallis tests indicated no statistically significant between-group differences for age, education, or monthly income for rs1360780 genotypes; all $p s>0.05$ (see Table 1). Overall, participants had experienced a range of 0-12 traumatic events in their lifetime, with a mode of 3 , median of 4 , and mean of 4.6 events, according to the TEI (total score). Lifetime trauma exposure did not correspond with FA in the left or right PC (study-defined mask), as indicated by a correlational analysis (left PC FA: $r=-0.06, p=0.59$; right PC FA: $r=0.05, p=0.64)$ as well as a two-tailed $t$-test comparing individuals with high and low trauma exposure, categorized using a median split of TEI total (left PC FA: $t=1.2, p=0.23$; right PC FA: $t=-0.47, p=0.64)$. Participants endorsed total PSS scores of $0-40$, with a mode of 0 , median of 11 , and mean of 14; a total of 34 participants met diagnostic criteria for PTSD. Results from the TEI indicated that trauma rates were comparable between the genotype groups; on average, participants had witnessed, experienced, or been confronted with approximately four Criterion A equivalent traumatic incidents of various types (eg, witnessing gun violence, being sexually assaulted). There were no significant between-group differences in trauma exposure measured across the lifespan (TEI total score) for adulthood (TEI adult trauma) or childhood maltreatment (CTQ sexual abuse, physical abuse, emotional abuse, and total score); all $p s>0.05$. There were no significant between-group differences in total PTSD symptoms (PSS total score; $p>0.05$ ).

\section{Associations between FKBP5 Genotype and Posterior Cingulum Integrity}

Univariate ANCOVA results indicated that, after statistically controlling for variance associated with age, PTSD symptoms, childhood maltreatment, and adult trauma exposure, significant between-group differences in the rs1360780 genotype were observed for FA in the left PC, as defined by our own mask $\left(F_{2,73}=6.96 ; p=0.002\right.$; see Figure 2$)$; in this model, left PC FA was significantly associated with sexual abuse (CTQ sexual abuse; $F_{2,73}=5.5 ; p=0.02$ ) but not with the other covariates, including other childhood abuse, PTSD symptoms, and trauma exposure in adulthood (all $p s>0.05$ ). These findings were confirmed in an ANCOVA conducted with left PC FA values extracted from an atlas-based mask $\left(\mathrm{F}_{2,73}=3.59 ; p=0.03\right)$; in this model, rs1360780 genotype was significantly associated with sexual abuse (CTQ sexual abuse; $F_{2,73}=5.5 ; p=0.02$ ), as well as trauma exposure in adulthood (TEI adult trauma total; $\mathrm{F}_{2,73}=4.5 ; p=0.04$ ).

Tukey's post-hoc tests indicated that left PC FA (defined by our mask) was significantly higher in the CC genotype (ie, participants with no copies of the risk allele; mean $=0.46, \mathrm{SD}=0.06)$ compared with the TT genotype group (ie, participants with two copies of the risk allele; mean $=0.41, S D=0.05)$. Results of a separate univariate ANCOVA demonstrated no between-group differences in rs1360780 genotype for right PC FA, although a nonsignificant trend emerged with our right PC mask $\left(\mathrm{F}_{2,73}=2.17 ; p=0.12\right)$ and an atlas-based mask of the right $\mathrm{PC}\left(\mathrm{F}_{2,73}=1.75 ; p=0.18\right)$.

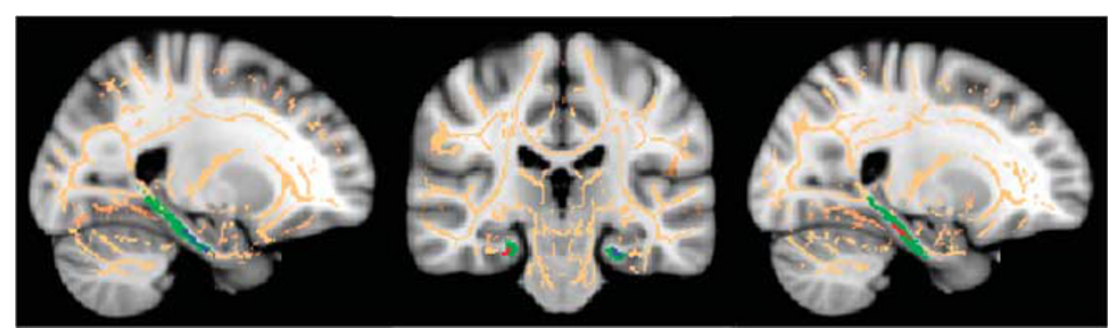

Figure I Masks of the posterior cingulum. Masks of the left (shown in blue) and right (shown in red) posterior cingulum overlaid on the MNI standard brain, displayed in radiological convention. Green voxels depict masks created using the Johns Hopkins University White Matter Tractography Atlas. Orange voxels depict mean FA skeleton for the entire sample. 


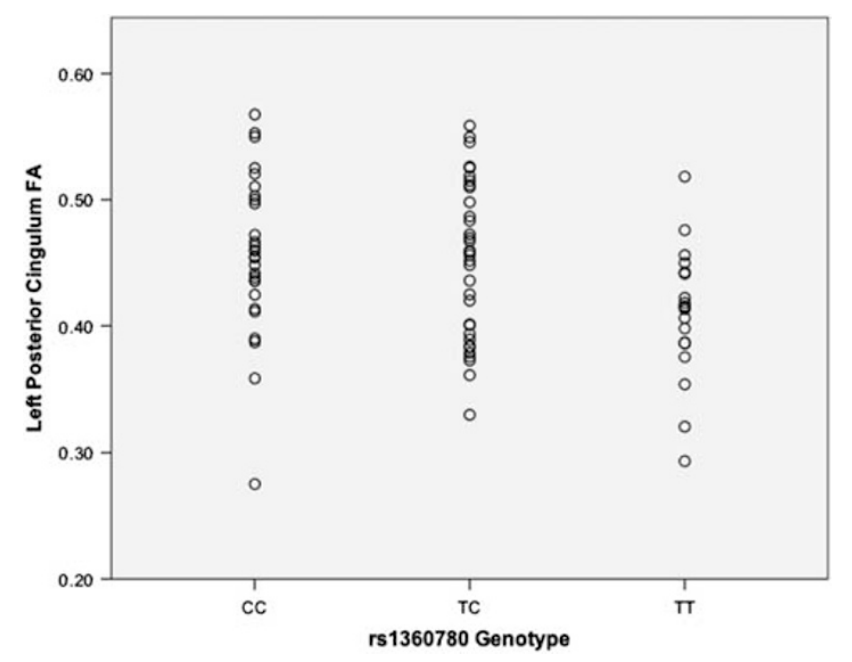

Figure 2 Fractional anisotropy in the left posterior cingulum and FKBP5 genotype. Lower fractional anisotropy in the left posterior cingulum observed for FKBP5 risk allele carriers (SNP rs I 360780; T = risk allele).

Factorial ANCOVAs were also used to test FKBP5 genotype by PTSD diagnosis interactions on left PC FA. No significant interactions between genotype and diagnosis were observed; however, compared with PTSD diagnosis and covariates, rs1360780 genotype demonstrated the largest (and most statistically significant) effect size in the overall models for left PC FA for both our mask $\left(\eta_{p}^{2}=0.15\right.$; see Supplementary Table S1) as well as FA for an atlas-based mask of the left PC $\left(\eta_{p}^{2}=0.1\right.$; see Supplementary Table S2).

Factorial ANCOVA results indicated no significant interactions of genotype and abuse history (presence or absence of emotional, physical, or sexual abuse, measured with the CTQ) for left PC FA $\left(\mathrm{F}_{2,73}=0.64 ; p=0.53\right)$ or right PC FA $\left(\mathrm{F}_{2,73}=0.1 ; p=0.91\right)$ after statistically controlling for variance associated with age, PTSD symptoms, and trauma exposure in adulthood. Factorial ANCOVA findings were similar for left PC FA $\left(\mathrm{F}_{2,73}=1.6 ; p=0.21\right)$ and right PC FA $\left(\mathrm{F}_{2,73}=0.24 ; p=0.79\right)$ as defined by an atlas.

\section{DISCUSSION}

The primary objective of this study was to investigate FKBP5 genotype associations with microstructural integrity of the PC in a sample of individuals with varying degrees of trauma and PTSD symptoms. For rs1360780, a putatively functional FKBP5 SNP, we observed that carriers of two risk alleles demonstrated significantly lower FA in the left PC compared with individuals with no risk alleles. In our previous, brain-wide study of white matter integrity in trauma survivors, we found that individuals with PTSD demonstrated lower FA in the PC, compared with controls that were matched for trauma exposure (Fani et al, 2012); in this analysis, the left PC demonstrated more significant differences in FA between groups as compared with the right PC, as indicated by the cluster size. Given that the two groups had experienced relatively similar degrees of psychological adversity, we hypothesized that genetic substrates may, at least in part, account for the FA differences that were observed. The present findings confirm this hypothesis, indicating that, compared with non-risk allele carriers, participants with two risk alleles for this SNP demonstrate decrements in the left PC microarchitecture. These findings were significant even after controlling for factors that may adversely influence PC FA, such as age, trauma exposure, and PTSD symptomatology; compared with these other factors, FKBP5 genotype demonstrated the largest effect size in our statistical models. These findings suggest that the abnormalities in left PC integrity observed here are more relevant to FKBP5 genotypic status than aging, trauma exposure, or PTSD sequelae.

Thus, the lower PC FA found in FKBP5 risk allele carriers may represent an intermediate neural phenotype for psychiatric disorders such as PTSD-a discrete, heritable white matter profile that has been associated with disease phenotype. The ostensibly small differences in PC FA between the different FKBP5 genotype groups (which may reflect lower myelination of this white matter tract in the risk group) are likely to have significant functional consequences, with regard to both cognition and emotion. In support of this notion, recent studies involving training on a mental or physical task have indicated that even relatively small changes in diffusion indices (on the order of $\sim 0.02$ ) correspond with differences in performance following training (Mackey et al, 2012; Scholz et al, 2009). Thus, the PC abnormalities found in FKBP5 risk allele carriers may represent a heritable source of vulnerability for the development of emotional and cognitive difficulties that characterize anxious and depressive pathology. In the presence of extreme psychological stress, individuals with this genetic profile may be at significantly higher risk for developing psychiatric disorders such as PTSD via alterations in PC integrity.

To our knowledge, this is the first study to demonstrate a link between FKBP5 polymorphisms and white matter integrity, or, more specifically, the PC, a white matter tract adjacent to the hippocampus. As noted earlier, FKBP5 is highly expressed in the hippocampus (Scharf et al, 2011) and appears to influence hippocampal function and morphology (Fani et al, 2013) possibly via alterations in GR sensitivity. The present data indicate that FKBP5 may also influence integrity of the PC, a white matter tract that is a branch of the larger cingulum bundle, which facilitates communication between the hippocampus and the cingulate gyrus. Numerous cognitive processes that engage the hippocampus and ACC may be affected by compromised PC integrity. Earlier studies have shown that PC integrity corresponds significantly with verbal (Delano-Wood et al, 2012) and visual memory abilities (Kantarci et al, 2011), as well as executive processes (Schermuly et al, 2010) such as the suppression of pre-potent response, as required by the Stroop task (Metzler-Baddeley et al, 2012). Notably, impairments in these processes characterize anxious individuals (Bremner et al, 2004; Gilbertson et al, 2001; Vasterling et al, 1998; Vasterling et al, 2002; Yehuda et al, 1995). The present findings suggest that FKBP5 polymorphisms may influence the development of a white matter tract, the left PC, and that integrity of this tract may be directly relevant to the etiology of these cognitive impairments.

These FKBP5-related alterations in left PC integrity are also likely to have a role in the development of anxiety symptoms, possibly via effects on fear-extinction processes. Extinction is a type of learning that occurs when a neutral 
cue that was previously paired with an aversive stimulus appears repeatedly in the absence of the aversive stimulus; during extinction, the defensive physiological response is attenuated. The PC represents a primary route of communication between the hippocampus and cingulate cortex, regions involved with learning to inhibit and extinguish fearful responses to threat cues. By exerting effects on this white matter tract, FKBP5 may indirectly influence extinction processes and thus affect risk for anxiety disorders, such as PTSD. Links between PC FA and PTSD have been observed in earlier studies of traumatized populations (Fani et al, 2012; Wang et al, 2010); in light of these findings, it is possible that FKBP5 influences a white matter diathesis for the later development of pathological anxiety or fear responses.

Of note is the fact that our current findings were localized to the left hemisphere; no significant associations were observed with FA in the right PC. The cause for the laterality of our findings remains unclear, given that there are no apparent theoretical reasons for these results. Other studies have identified lower FA in the left, but not the right cingulum, in trauma survivors both with and without PTSD (Choi et al, 2009; Kim et al, 2006); in light of these data, it is possible that the integrity of PC in the left hemisphere is more susceptible to the effects of extreme psychological stress. However, the results of another study implicates the right PC (Wang et al, 2010); therefore, hypotheses regarding laterality must be tested in further studies.

Several limitations must be noted regarding the methodology of the present study. Given that this was a crosssectional study that included chronically traumatized and pathologically anxious individuals, it is not possible to fully extricate genotype effects from the sequelae of environmental adversity or post-traumatic psychopathology. Although not all participants had experienced psychological trauma, most participants had experienced one or more traumatic events, thus it is possible that these findings may be more specific to trauma-exposed populations. In addition, given that this was a single candidate gene study, it is likely that polymorphisms in other genes also contribute to the degradations in PC FA observed here. Although we observed main effects of FKBP5 genotype, no significant interaction between genotype and PTSD was observed; given that only 20 participants had two copies of the risk allele, insufficient statistical power may have interfered with our ability to detect this interaction. We neither observed significant associations between FKBP5 genotype and PTSD nor any significant interactions of genotype and childhood maltreatment, unlike some previous gene-disease association studies (Binder et al, 2008; Xie et al, 2010). However, the sample size of the present study is significantly smaller than these studies. Further, although our earlier study indicated clear associations between PTSD and PC FA (Fani et al, 2012), the associations between PTSD and PC FA only approached significance in our statistical models (eg, $p=0.07$, see Supplementary Table S2); this may also be due to insufficient statistical power, as the presence and severity of PTSD symptoms in the present sample was lower than our earlier study. Possibly, our study findings support the notion that lower FA in the PC represents an intermediate phenotype for anxious psychopathology in general and that these findings are not exclusive to PTSD; this hypothesis also warrants investigation in further research.

In conclusion, we observed an association between FKBP5 polymorphisms and white matter integrity, specifically, microstructural integrity of the left PC. Individuals who carried two risk alleles for polymorphisms of this gene demonstrated comparatively lower FA in this white matter tract than those who carried one or no risk alleles, and this relationship was significant even after accounting for variance associated with age, trauma exposure, and PTSD symptoms. These data highlight the possibility that specific FKBP5 genotypes may increase risk for anxiety disorders via alterations in white matter integrity. Following psychological trauma, alterations in the left PC microarchitecture could give rise to some of the cognitive and emotional problems that characterize pathological anxiety. Thus, the present data support the possibility that decrements in left PC FA represent an intermediate phenotype for psychiatric disorders, such as PTSD. Further studies of FKBP5 associations with PC integrity in populations with other mood and anxiety symptoms are warranted to extend these findings to other psychiatric disorders.

\section{FUNDING AND DISCLOSURE}

This work was primarily supported by the National Institutes of Mental Health (R01 MH071537, M01RR00039, and P20RR16435 to KJR, F32MH095456 to NF). Support was also received from the Howard Hughes Medical Institute, PHS Grant UL1 RR025008 from the Clinical and Translational Science Award program, National Center for Research Resources, and the Burroughs Wellcome Fund (KJR). The authors declare no conflict of interest.

\section{ACKNOWLEDGEMENTS}

We thank the participants who made this work possible, as well as the staff of the Grady Trauma Project, particularly Allen W. Graham, Angelo Brown, Amreen Dharani, Jennifer S. Davis, Jennifer Winkler, Sarah Spann, and Andrew Pallos. We wish to thank Jaemin Shin and Timothy Ely for their valuable assistance with data analysis, and the Georgia State University-Georgia Institute of Technology Center for Advanced Brain Imaging for data support.

\section{REFERENCES}

Acheson DT, Gresack JE, Risbrough VB. (2012). Hippocampal dysfunction effects on context memory: possible etiology for posttraumatic stress disorder. Neuropharmacology 62: 674-685.

Almli LM, Fani N, Smith AK, Ressler KJ. (2013). Genetic approaches to understanding post-traumatic stress disorder. Int J Neuropsychopharmacol (e-pub ahead of print 8 October 2013).

Alonso G (2000). Prolonged corticosterone treatment of adult rats inhibits the proliferation of oligodendrocyte progenitors present throughout white and gray matter regions of the brain. Glia 31: 219-231.

Bernstein DP, Fink L. (1998). Childhood Trauma Questionnaire: a Retrospective Self-Report Manual. The Psychological Corporation: New York, NY, USA. 
Bevilacqua L, Carli V, Sarchiapone M, George DK, Goldman D, Roy A et al (2012). Interaction between FKBP5 and childhood trauma and risk of aggressive behavior. Arch Gen Psychiatry 69: 62-70.

Binder EB (2009). The role of FKBP5, a co-chaperone of the glucocorticoid receptor in the pathogenesis and therapy of affective and anxiety disorders. Psychoneuroendocrinology 34(Suppl 1): S186-S195.

Binder EB, Bradley RG, Liu W, Epstein MP, Deveau TC, Mercer KB et al (2008). Association of FKBP5 polymorphisms and childhood abuse with risk of posttraumatic stress disorder symptoms in adults. JAMA 299: 1291-1305.

Boscarino JA, Erlich PM, Hoffman SN, Zhang X. (2012). Higher FKBP5, COMT, CHRNA5, and CRHR1 allele burdens are associated with PTSD and interact with trauma exposure: implications for neuropsychiatric research and treatment. Neuropsychiatr Dis Treat 8: 131-139.

Bradley RG, Binder EB, Epstein MP, Tang Y, Nair HP, Liu W et al (2008). Influence of child abuse on adult depression: moderation by the corticotropin-releasing hormone receptor gene. Arch Gen Psychiatry 65: 190-200.

Bremner JD, Randall P, Scott TM, Bronen RA, Seibyl JP, Southwick SM et al (1995). MRI-based measurement of hippocampal volume in patients with combat-related posttraumatic stress disorder. Am J Psychiatry 152: 973-981.

Bremner JD, Vermetten E, Afzal N, Vythilingam M. (2004). Deficits in verbal declarative memory function in women with childhood sexual abuse-related posttraumatic stress disorder. J Nerv Ment Dis 192: 643-649.

Bremner JD, Vythilingam M, Vermetten E, Southwick SM, McGlashan T, Nazeer A et al (2003). MRI and PET study of deficits in hippocampal structure and function in women with childhood sexual abuse and posttraumatic stress disorder. Am J Psychiatry 160: 924-932.

Chen L, Chen X, Liu W, Wang Q, Jiang T, Wang J et al (2013). White matter microstructural abnormalities in patients with late-onset schizophrenia identified by a voxel-based diffusion tensor imaging. Psychiatry Res 212: 201-207.

Chiang MC, Avedissian C, Barysheva M, Toga AW, McMahon KL, de Zubicaray GI et al (2009). Extending genetic linkage analysis to diffusion tensor images to map single gene effects on brain fiber architecture. Med Image Comput Comput Assist Interv 12(Pt 2): 506-513.

Choi J, Jeong B, Rohan ML, Polcari AM, Teicher MH (2009). Preliminary evidence for white matter tract abnormalities in young adults exposed to parental verbal abuse. Biol Psychiatry 65: 227-234.

Collip D, Myin-Germeys I, Wichers M, Jacobs N, Derom C, Thiery $\mathrm{E}$ et al (2013). FKBP5 as a possible moderator of the psychosisinducing effects of childhood trauma. $\mathrm{Br} J$ Psychiatry 202: 261-268.

Delano-Wood L, Stricker NH, Sorg SF, Nation DA, Jak AJ, Woods SP et al (2012). Posterior cingulum white matter disruption and its associations with verbal memory and stroke risk in mild cognitive impairment. J Alzheimers Dis 29: 589-603.

Drago V, Babiloni C, Bartres-Faz D, Caroli A, Bosch B, Hensch T et al (2011). Disease tracking markers for Alzheimer's disease at the prodromal (MCI) stage. J Alzheimers Dis 26(Suppl 3): 159-199.

Falsetti SA, Resnick HS, Resick PA, Kilpatrick DG. (1993). The modified PTSD symptom scale: a brief self-report measure of posttraumatic stress disorder. Behav Ther 16: 161-162.

Fani N, Gutman D, Tone EB, Almli L, Mercer KB, Davis J et al (2013). FKBP5 and attention bias for threat: associations with hippocampal function and shape. JAMA Psychiatry 70: 392-400.

Fani N, King TZ, Jovanovic T, Glover EM, Bradley B, Choi K et al (2012). White matter integrity in highly traumatized adults with and without post-traumatic stress disorder. Neuropsychopharmacology 37: 2740-2746.
Fujiwara H, Namiki C, Hirao K, Miyata J, Shimizu M, Fukuyama H et al (2007). Anterior and posterior cingulum abnormalities and their association with psychopathology in schizophrenia: a diffusion tensor imaging study. Schizophr Res 95: 215-222.

Gilbertson MW, Gurvits TV, Lasko NB, Orr SP, Pitman RK. (2001). Multivariate assessment of explicit memory function in combat veterans with posttraumatic stress disorder. J Trauma Stress 14: 413-432.

Gurvits TV, Shenton ME, Hokama H, Ohta H, Lasko NB, Gilbertson MW et al (1996). Magnetic resonance imaging study of hippocampal volume in chronic, combat-related posttraumatic stress disorder. Biol Psychiatry 40: 1091-1099.

Hua K, Zhang J, Wakana S, Jiang H, Li X, Reich DS et al (2008). Tract probability maps in stereotaxic spaces: analyses of white matter anatomy and tract-specific quantification. Neuroimage 39: 336-347.

Ising M, Depping AM, Siebertz A, Lucae S, Unschuld PG, Kloiber S et al (2008). Polymorphisms in the FKBP5 gene region modulate recovery from psychosocial stress in healthy controls. Eur $J$ Neurosci 28: 389-398.

Jenkinson M, Smith S. (2001). A global optimisation method for robust affine registration of brain images. Med Image Anal 5: 143-156.

Kantarci K, Senjem ML, Avula R, Zhang B, Samikoglu AR, Weigand SD et al (2011). Diffusion tensor imaging and cognitive function in older adults with no dementia. Neurology 77: 26-34.

Kazlouski D, Rollin MD, Tregellas J, Shott ME, Jappe LM, Hagman JO et al (2011). Altered fimbria-fornix white matter integrity in anorexia nervosa predicts harm avoidance. Psychiatry Res 192: $109-116$.

Kim SJ, Jeong DU, Sim ME, Bae SC, Chung A, Kim MJ et al (2006). Asymmetrically altered integrity of cingulum bundle in posttraumatic stress disorder. Neuropsychobiology 54: 120-125.

Klengel T, Mehta D, Anacker C, Rex-Haffner M, Pruessner JC, Pariante CM et al (2013). Allele-specific FKBP5 DNA demethylation mediates gene-childhood trauma interactions. Nat Neurosci 16: $33-41$.

Kochunov P, Glahn DC, Lancaster JL, Winkler AM, Smith S, Thompson PM et al (2010). Genetics of microstructure of cerebral white matter using diffusion tensor imaging. Neuroimage 53: 1109-1116.

Lavebratt C, Aberg E, Sjoholm LK, Forsell Y. (2010). Variations in FKBP5 and BDNF genes are suggestively associated with depression in a Swedish population-based cohort. J Affect Disord 125: 249-255.

Lebel C, Gee M, Camicioli R, Wieler M, Martin W, Beaulieu C. (2012). Diffusion tensor imaging of white matter tract evolution over the lifespan. Neuroimage 60: 340-352.

Luijk MP, Velders FP, Tharner A, van Ijzendoorn MH, BakermansKranenburg MJ, Jaddoe VW et al (2010). FKBP5 and resistant attachment predict cortisol reactivity in infants: gene-environment interaction. Psychoneuroendocrinology 35: 1454-1461.

Ly M, Canu E, Xu G, Oh J, McLaren DG, Dowling NM et al (2013). Midlife measurements of white matter microstructure predict subsequent regional white matter atrophy in healthy adults. Hum Brain Mapp (e-pub ahead of print 17 July 2013; doi:10.1002/hbm.22311).

Mackey AP, Whitaker KJ, Bunge SA. (2012). Experience-dependent plasticity in white matter microstructure: reasoning training alters structural connectivity. Front Neuroanat 6: 32.

Mehta D, Gonik M, Klengel T, Rex-Haffner M, Menke A, Rubel J et al (2011). Using polymorphisms in FKBP5 to define biologically distinct subtypes of posttraumatic stress disorder: evidence from endocrine and gene expression studies. Arch Gen Psychiatry 68: 901-910.

Metzler-Baddeley C, Jones DK, Steventon J, Westacott L, Aggleton JP, O'Sullivan MJ. (2012). Cingulum microstructure predicts 
cognitive control in older age and mild cognitive impairment. J Neurosci 32: 17612-17619.

Morimoto M, Morita N, Ozawa H, Yokoyama K, Kawata M (1996). Distribution of glucocorticoid receptor immunoreactivity and mRNA in the rat brain: an immunohistochemical and in situ hybridization study. Neurosci Res 26: 235-269.

Paillere Martinot ML, Lemaitre H, Artiges E, Miranda R, Goodman $\mathrm{R}$, Penttila J et al (2013). White-matter microstructure and graymatter volumes in adolescents with subthreshold bipolar symptoms. Mol Psychiatry (e-pub ahead of print 30 April 2013; doi:10.1038/mp.2013.44).

Scharf SH, Liebl C, Binder EB, Schmidt MV, Muller MB (2011). Expression and regulation of the Fkbp5 gene in the adult mouse brain. PLoS One 6: e16883.

Schermuly I, Fellgiebel A, Wagner S, Yakushev I, Stoeter P, Schmitt $\mathrm{R}$ et al (2010). Association between cingulum bundle structure and cognitive performance: an observational study in major depression. Eur Psychiatry 25: 355-360.

Scholz J, Klein MC, Behrens TE, Johansen-Berg H. (2009). Training induces changes in white-matter architecture. Nat Neurosci 12: 1370-1371.

Shin LM, Liberzon I. (2010). The neurocircuitry of fear, stress, and anxiety disorders. Neuropsychopharmacology 35: 169-191.

Smith SM, Jenkinson M, Johansen-Berg H, Rueckert D, Nichols TE, Mackay CE et al (2006). Tract-based spatial statistics: voxelwise analysis of multi-subject diffusion data. Neuroimage 31: 1487-1505.

Smith SM, Jenkinson M, Woolrich MW, Beckmann CF, Behrens TE, Johansen-Berg $\mathrm{H}$ et al (2004). Advances in functional and structural MR image analysis and implementation as FSL. Neuroimage 23(Suppl 1): S208-S219.

Smith SM. (2002). Fast robust automated brain extraction. Hum Brain Mapp 17: 143-155.

Sylvester CM, Corbetta M, Raichle ME, Rodebaugh TL, Schlaggar BL, Sheline YI et al (2012). Functional network dysfunction in anxiety and anxiety disorders. Trends Neurosci 35: 527-535.

Thomason ME, Thompson PM (2011). Diffusion imaging, white matter, and psychopathology. Annu Rev Clin Psychol 7: 63-85.
Tromp do PM, Grupe DW, Oathes DJ, McFarlin DR, Hernandez PJ, Kral TR et al (2012). Reduced structural connectivity of a major frontolimbic pathway in generalized anxiety disorder. Arch Gen Psychiatry 69: 925-934.

Vasterling JJ, Brailey K, Constans JI, Sutker PB. (1998). Attention and memory dysfunction in posttraumatic stress disorder. Neuropsychology 12: 125-133.

Vasterling JJ, Duke LM, Brailey K, Constans JI, Allain AN Jr., Sutker PB. (2002). Attention, learning, and memory performances and intellectual resources in Vietnam veterans: PTSD and no disorder comparisons. Neuropsychology 16: 5-14.

Wang $\mathrm{HH}$, Zhang ZJ, Tan QR, Yin $\mathrm{H}$, Chen YC, Wang $\mathrm{HN}$ et al (2010). Psychopathological, biological, and neuroimaging characterization of posttraumatic stress disorder in survivors of a severe coalmining disaster in China. J Psychiatr Res 44: 385-392.

Xie P, Kranzler HR, Poling J, Stein MB, Anton RF, Farrer LA et al (2010). Interaction of FKBP5 with childhood adversity on risk for post-traumatic stress disorder. Neuropsychopharmacology 35: $1684-1692$.

Yehuda R, Keefe RS, Harvey PD, Levengood RA, Gerber DK, Geni J et al (1995). Learning and memory in combat veterans with posttraumatic stress disorder. Am J Psychiatry 152: 137-139.

Zeng LL, Shen H, Liu L, Wang L, Li B, Fang P et al (2012). Identifying major depression using whole-brain functional connectivity: a multivariate pattern analysis. Brain $135(\mathrm{Pt} 5)$ : $1498-1507$.

Zhang J, Wang J, Wu Q, Kuang W, Huang X, He Y et al (2011). Disrupted brain connectivity networks in drug-naive, first-episode major depressive disorder. Biol Psychiatry 70: 334-342.

Zhang Y, Schuff N, Jahng GH, Bayne W, Mori S, Schad L et al (2007). Diffusion tensor imaging of cingulum fibers in mild cognitive impairment and Alzheimer disease. Neurology 68: 13-19.

Zobel A, Schuhmacher A, Jessen F, Hofels S, von Widdern O, Metten $\mathrm{M}$ et al (2010). DNA sequence variants of the FKBP5 gene are associated with unipolar depression. Int J Neuropsychopharmacol 13: 649-660.

Supplementary Information accompanies the paper on the Neuropsychopharmacology website (http://www.nature.com/npp) 